\title{
Interfaced SEM and micro-Raman Spectroscopy for SERS Analysis of Dyes on Single Fibers
}

\author{
Sergey V. Prikhodko ${ }^{1}$, Diana C. Rambaldi ${ }^{2}$, Vanessa Muros $^{3}$, Elizabeth Burr ${ }^{3}$, and Ioanna Kakoulli ${ }^{1,3}$ \\ ${ }^{1}$ Department of Materials Science and Engineering, UCLA, Los Angeles, CA 90095-1595. \\ ${ }^{2}$ Conservation Center, Los Angeles County Museum of Art, Los Angeles, CA 90036. \\ ${ }^{3}$ UCLA/Getty Conservation Program, Cotsen Institute of Archaeology, UCLA, Los Angeles, CA 90095-1510.
}

The identification of dyestuff in ancient textiles often provides a major analytical challenge. High Performance Liquid Chromatography (HPLC) coupled with diode-array detection (DAD) and/or mass spectrometry (MS) is the most used technique for the identification of dye molecules. However, this technique has severe limitations in the study of ancient dyed fibers due to the large amount of sample required [1]. Moreover, chemical extraction processes necessary for HPLC analysis can be detrimental to these severely size-limited and irreplaceable archaeological materials.

To overcome these analytical challenges, a system interfacing scanning electron microscopy and micro-Raman spectroscopy (SEM- $\mu \mathrm{RS}$ ) was used to develop an improved extractionless procedure for the identification of dyes on single fibers. For this non-destructive approach based on Surface-Enhanced Raman Scattering (SERS) detection, a small segment of a single fiber was treated with a silver nanocolloid adapted from the procedure described by Lee et al. [2]. When stimulated by a laser beam, these silver nanoparticles (AgNPs) not only enhance the Raman signals of several orders of magnitude [3,4], but also quench molecular fluorescence improving the signal to noise ratio.

Here we show the SERS results using variable pressure SEM, Nova 230 (FEI), interfaced with confocal Raman system, in-Via (Renishaw) uses $785 \mathrm{~nm}$ laser probe, for the analysis of two different dyed fibers. The first is an alpaca fiber dyed with Peruvian cochineal without any mordant (Figure 1). The second is a wool fiber dyed with Indian madder (Figure 2) from the Schweppe collection of yarns (courtesy of the Getty Conservation Institute). The wool fiber was mordanted with $25 \%$ alum and $6 \%$ tartar. The two dyed fibers were selected to show the ability of this method to distinguish between different dyestuff molecules used at very small concentrations.

The selection of areas to be analyzed with the $\mu$-RS was significantly improved by using SEM (Figures 1A) rather than traditional optical microscopy (Figure 1B). SEM images showed that when metal nanocolloids are deposited on the fiber surface to enable SERS analysis, the distribution of AgNPs is not uniform and the thickness of deposited layer may vary largely. The results showed that the analysis of areas with a thin layer of deposited AgNPs led to optimal reproducible spectra (Figure 1C, and [3]). On the contrary, areas with a thick layer of AgNPs (Figure 1A, spot 2) or without AgNPs deposition (Figure 2A, spot 2) resulted in a low signal to noise ratio (Figure 1C, spectrum [1]) or no signal at all (Figure 2C, spectrum [2]). The use of SEM thus proved to be fundamental to easily locate areas of thin and uniform AgNPs deposition enabling successful and reproducible SERS analysis.

[1] X.Zhang, et al. Anal Chem 79 (2007) 1575-1582.

[2] P.C.Lee, et al. J Phys Chem 86 (1982) 3391-3395

[3] Z.Jurasekova, et al. J Raman Spectrosc 41 (2010) 1455-1461.

[4] B.Sharma, et al. Materials Today 15 (2012) 16-25. 

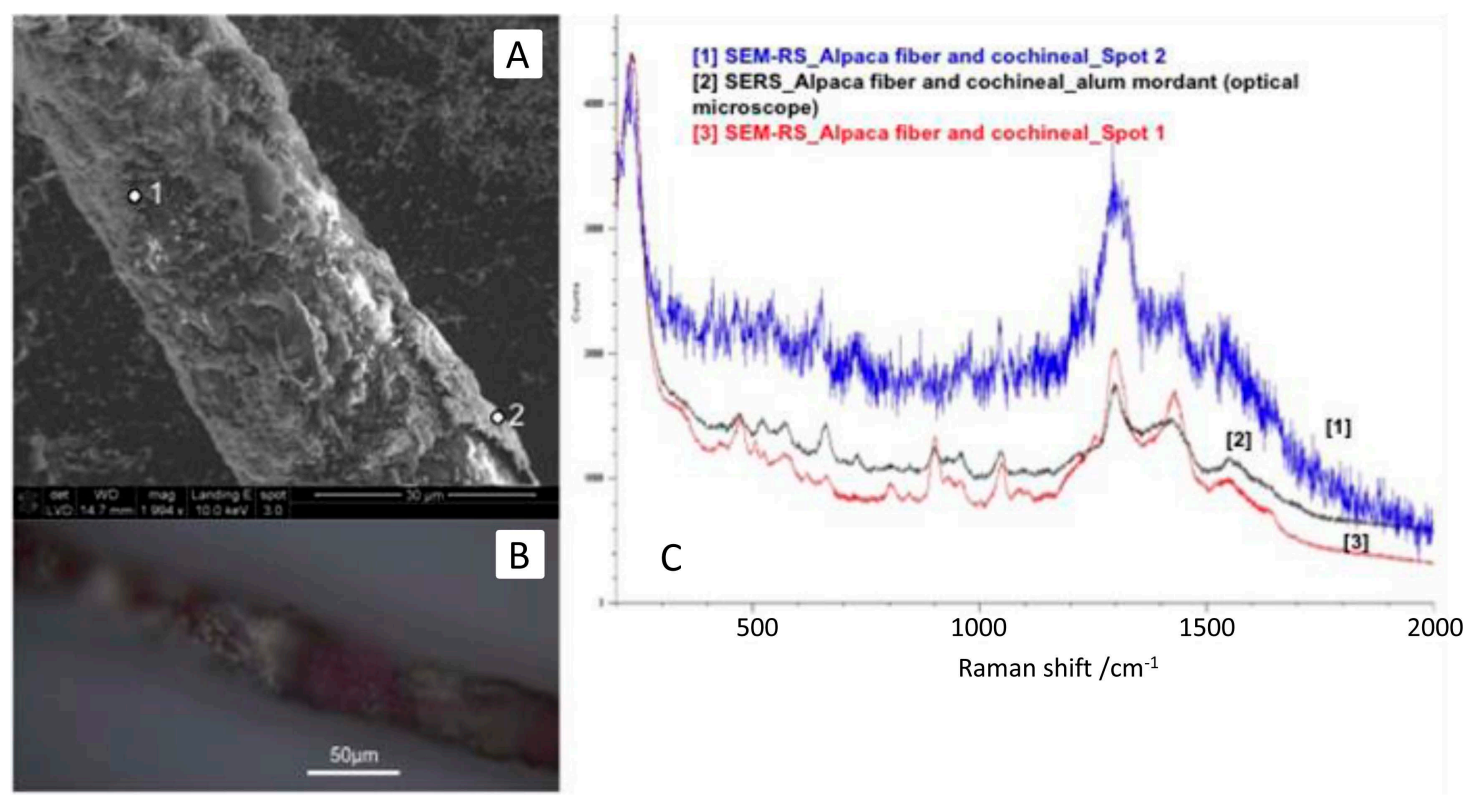

Fig.1 A. Secondary electron micrograph of alpaca fiber dyed with Peruvian cochineal. Spots 1 and 2 were analyzed using $\mu$-Raman spectroscopy. B. Optical image of the same fiber as in A. C. SERS spectra of: [1] alpaca fiber dyed with cochineal (spectrum acquired with the SEM- $\mu$ RS system in a spot with a thick layer of AgNPs, (Image A, spot 2)); [2] alpaca fiber dyed with cochineal mordanted with alum (spectrum acquired with a traditional $\mu$ RS presented for comparison purposes); [3] an alpaca fiber dyed with cochineal (spectrum acquired with the SEM- $\mu$ RS system in a spot covered with a uniform and thin layer of AgNPs (Image A, spot 1)).
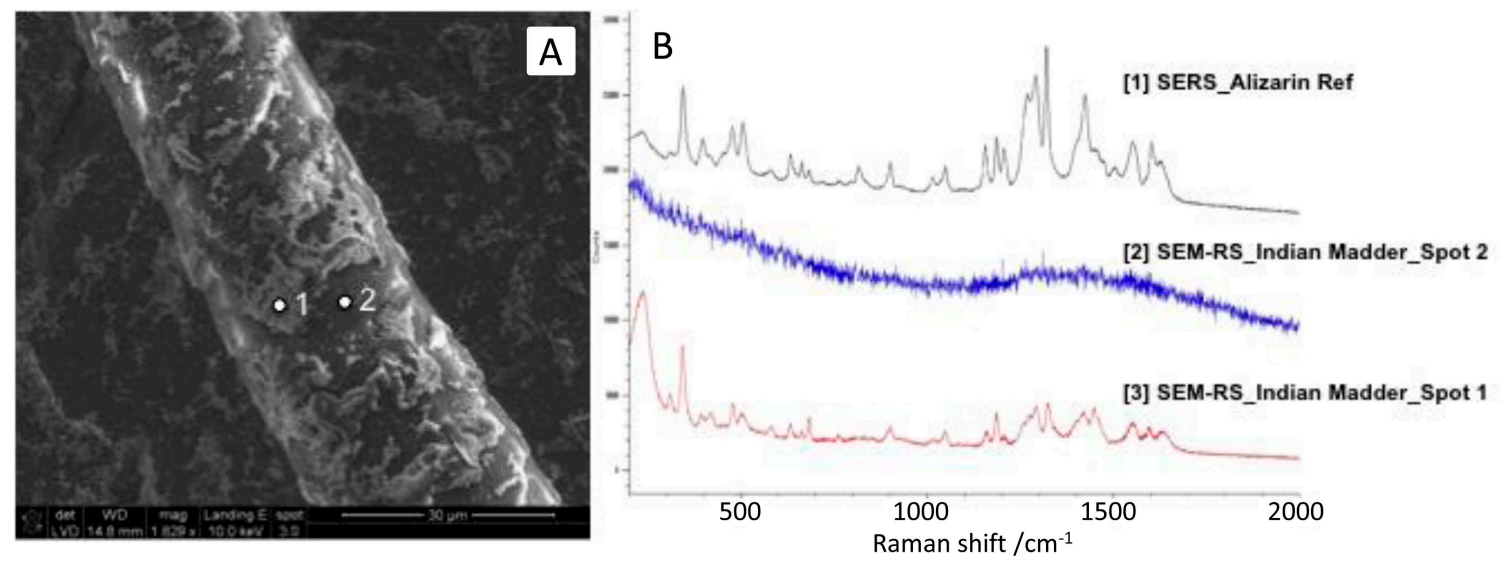

Fig.2 A. Secondary electron micrograph of wool fiber dyed with Indian madder and mordanted with alum and tartar. Spots 1 and 2 were analyzed using $\mu$-Raman spectroscopy. B. SERS spectra of: [1] standard alizarin - the main dyestuff found in Indian madder (the spectrum was acquired using a traditional $\mu \mathrm{RS}$ and is presented for comparison purposes); [2] wool fiber dyed with Indian madder (spectrum acquired with the SEM- $\mu \mathrm{RS}$ system in a spot without AgNPs deposited (Image A, spot 2)); [3] wool fiber dyed with Indian madder (spectrum acquired with the SEM- $\mu$ RS system in a spot with a thin deposition of AgNPs (Image A, spot 1)). 